\title{
versants
}

\section{«Credo che esista solo la letteratura». Conversazione con Olivo Barbieri}

\author{
Marco MAggi \& Vega Tescari \\ Università della Svizzera italiana \\ ORCID: 0ooo-0003-3292-776X, \\ oooo-0ool-7847-9208
}

\begin{abstract}
Conversazione con il fotografo Olivo Barbieri sullo statuto delle immagini e il suo rapporto con la parola e la letteratura. A partire da Flippers (19771978), proseguendo con la partecipazione a Viaggio in Italia (1984) e con le serie Artificial Illuminations (1980-2014), Virtual Truths (1996-2002), site specific_(2003-2013), Parks (2006-2015), Real Worlds (2008-2013), Olivo Barbieri conduce un'originale riflessione sulla quantità di realtà presente nei sistemi di vita contemporanei e su quanto la nostra percezione sia in grado di assorbirne. Presente con le sue opere nei principali musei e collezioni del mondo, è inoltre attento e appassionato curatore di cataloghi e libri fotografici.
\end{abstract}

Keywords: Olivo Barbieri, Letteratura e fotografia, Libro fotografico, Fototestualità.

M. M.: I suoi esordi sono legati a Viaggio in Italia (1984), un progetto al quale diede un contributo essenziale uno scrittore come Gianni Celati. Come si rapportava la sua fotografia con la scrittura in quella fase iniziale? C'era curiosità, oppure inibizione, soggezione?

O. B.: C'era molta curiosità. Gianni Celati lo conoscevo poco, non avevo letto molti suoi libri. Se un merito ha avuto Viaggio in Italia per quanto riguarda la fotografia è stato quello di redigere un piccolo regesto di chi fosse operativo in quegli anni. Di Gianni Celati mi piacque molto Finzioni occidentali. Mi sorprese che uno scrittore italiano si interessasse di fotografia contemporanea. Leggevo Alberto Arbasino.

In quegli anni mi interessavo moltissimo di letteratura. Ero attratto da scrittori come William Burroughs, che già alla fine degli anni Cinquanta sosteneva concetti innovativi: dobbiamo fare qualcosa, perché la letteratura ha quarant'anni di ritardo sulle arti figurative e il linguaggio è un virus.

Leggevo molto Gertrude Stein, per le sue relazioni con il Cubismo e con la nascita di ciò che ora definiamo arte moderna. Mi piaceva la letteratura sperimentale. 


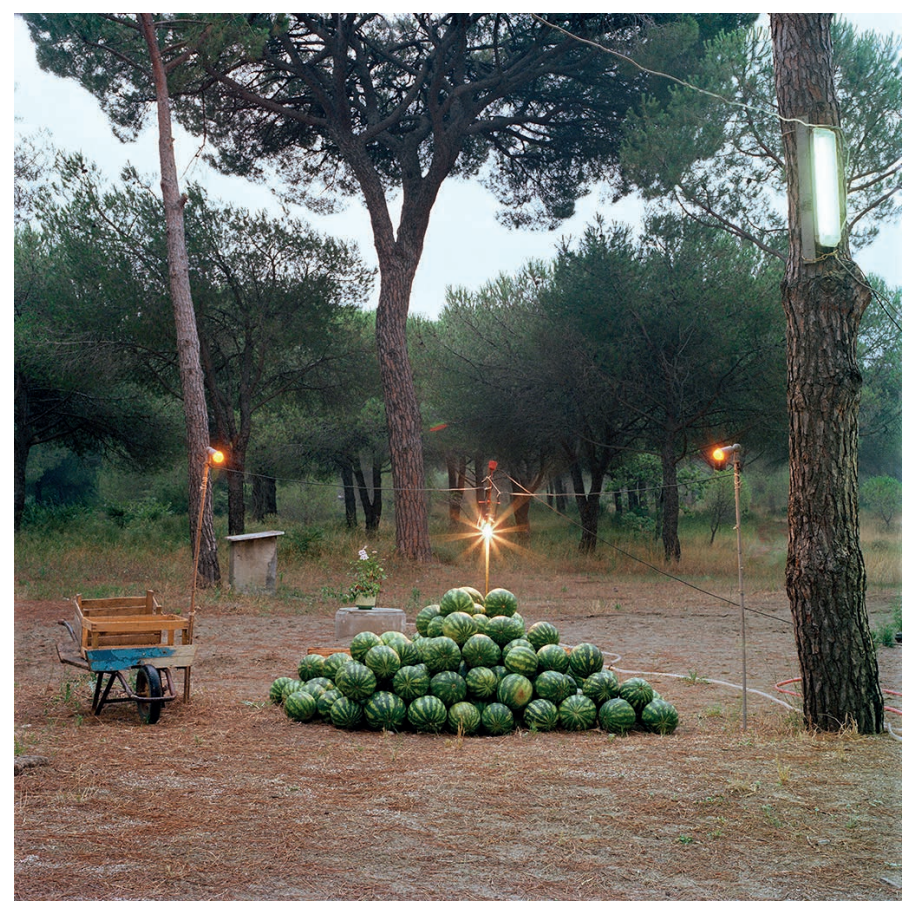

Fig. I: Olivo Barbieri, Follonica, Grosseto, I983.

V. T.: Ricordo un'intervista in cui lei cita Burroughs e richiama Flaubert, osservando come si tratti di un autore che i fotografi hanno poco considerato. Come descriverebbe la parabola del suo rapporto da fotografo con la parola e con la scrittura? Come è evoluto questo rapporto?

O. B.: Credo che se non avessi letto i Tre racconti di Gustave Flaubert e Tre esistenze di Gertrude Stein difficilmente avrei capito buona parte della fotografia contemporanea.

Donald Sutherland (omonimo dell'attore!), nel saggio Gertrude Stein: A biography of her work del I97I, da grecista spiega, partendo dalla cultura classica, la differenza tra lo spazio americano e quello europeo.

Senza nominarli fa capire l'importanza e la differenza di autori come Eugène Atget e Walker Evans. La Parigi di Atget e l'America visivamente inventata da Walker Evans, che tanto ha influenzato la mia generazione. La distanza dello spazio europeo era un enigma visivo da risolvere.

Le interviste di J. G. Ballard e Tristi Tropici e le interviste di Claude Lévi-Strauss sono state letture illuminanti per immaginare il futuro immediato. 
Verso i diciott'anni entrai in contatto con il mondo della fotografia italiana, che mi sembrò amatoriale e autoriferito. Abbandonai la fotografia e mi interessai di letteratura, però poi nel 1978 iniziai a esporre e ritornai alla fotografia.

La scrittura mi interessa ancora moltissimo. Credo però che lo studio delle immagini sia in grave ritardo, rispetto all'uso estremo che ne facciamo e per l'approssimativa conoscenza che ne abbiamo. Continuiamo a usare quasi solo parametri di studio antichi di cento anni, Aby Warburg e Walter Benjamin, l'onnipresente Marcel Duchamp. Tra noi e loro quasi solo Marshall McLuhan.

M. M.: A proposito delle tipologie di spazi cui faceva riferimento, un geografo, Matteo Meschiari, ha ragionato sulla differenza fra la casa-rifugio tipica dello spazio americano e la casa-magazzino/archivio tipica dello spazio europeo. Lei ha dedicato grande attenzione anche allo spazio americano, penso alla serie American Monument and Monument. In che modo si è rapportato a questo spazio, dopo il tentativo di costruire uno spazio europeo?

O. B.: Quasi tutti gli autori della mia generazione hanno abdicato all'idea di fotografare gli Stati Uniti, eravamo intimiditi dall'opera di Lee Friedlander e William Eggleston, per citarne solo due. Sembrava di entrare in un'area proibita, tutto era stato autorevolmente fatto e detto.

Ho fotografato gli Stati Uniti solo dopo aver avuto un'esperienza quasi trentennale in Cina. L'Occidente forse l'ho capito conoscendo l'Oriente. In termini di spazio, e forse di tempo, l'Occidente mi è diventato più intellegibile conoscendo l'Oriente. Nel primo catalogo che ho pubblicato sulla Cina, Appunti di viaggio in Cina del I989, ho scritto che c'è una differenza inesorabile tra il modo in cui tendiamo un filo noi e il modo in cui lo tendono i cinesi, anche semplicemente per stendere il bucato.

In quegli anni in Cina era in atto una rutilante e frenetica trasformazione architettonica e urbanistica. Cercavo di capire chiedendo a giornalisti e architetti quali fossero le influenze e secondo quali scelte si costruiva. Nel 1997 il famoso architetto Yung Ho Chang mi disse: «Gli immobiliaristi vanno in giro per il mondo, vedono edifici che li colpiscono, poi tornano e li mettono insieme disordinatamente, come se capricciosamente facessero shopping».

Nel 2005 in Tibet, per caso nell'hotel in cui alloggiavo incontrai Geremie Barmé, sinologo australiano di fama internazionale (ha realizzato documentari seminali sulla Rivoluzione culturale) ed è ritenuto uno dei capisaldi della sinologia occidentale. Ero appena tornato da Las Vegas, a cui avevo dedicato un libro, una mostra e un film. Barmé era con la fotografa americana Lois Conner per un progetto sul significato delle montagne in Cina. A me interessava vedere quanta Cina ci fosse in Tibet. Sfogliando il mio libro site specific_LAS VEGAS o5 fresco di stampa disse: «Queste immagini di- 
mostrano che per capire la Cina contemporanea devi conoscere Las Vegas». A seguito di questa riflessione nel 2017 ho realizzato American Monument and Monument, mettendo in relazione icone culturali e vernacolari. Le monumentali insegne delle ciambelle Donut e i dipinti di Mark Rothko. Da allora, spontaneamente, ho iniziato a decifrare aree del mondo come fossero parchi tematici, le grandi cascate, le grandi montagne, l'architettura cinese, le mille sfaccettature del web, le grandi discariche del sud est asiatico, i codici identitari.

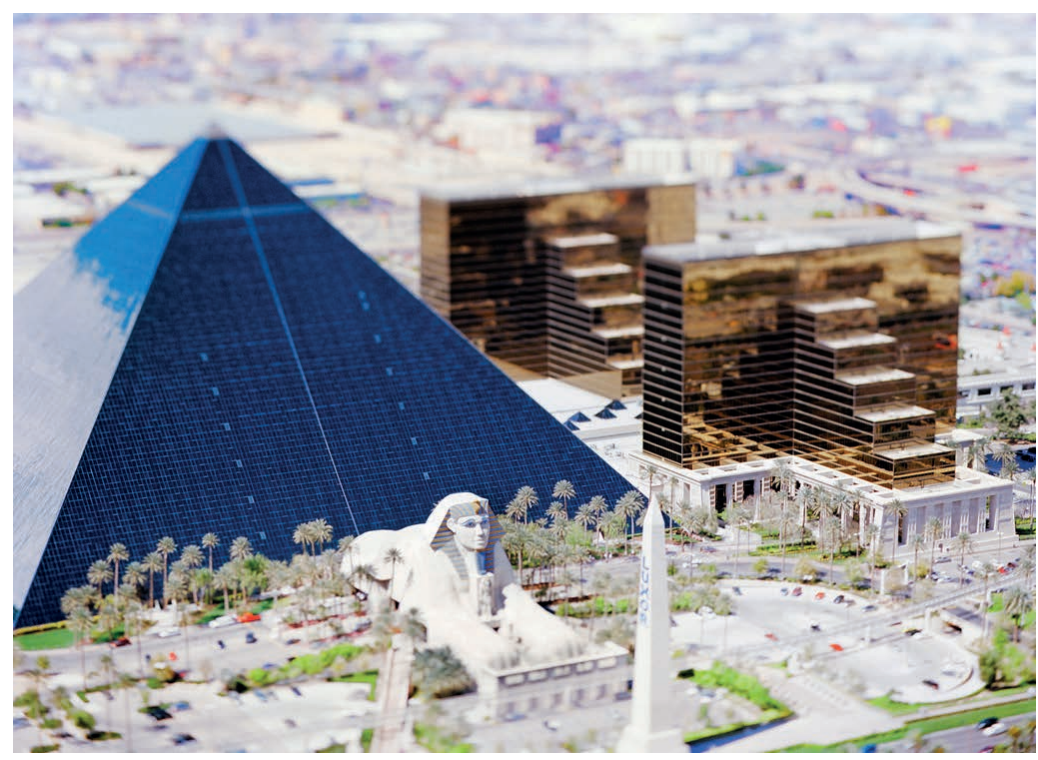

Fig. 2: Olivo Barbieri, site specific_LAS VEGAS 05, 2005.

V. T.: Lei ha realizzato molti libri fotografici, d'artista. Che importanza ha per lei l'architettura del libro, come costruisce l'oggetto-libro, quale attenzione gli riserva? Ci sono fotografi che scattano pensando alla dimensione espositiva, mentre ci sono fotografi come Luigi Ghirri che pensava, mentre scattava fotografie, alla forma-libro.

O. B.: I libri sono importanti perché rendono visibile l'intero progetto. A volte le mostre possono contenere solo poche opere. Il rapporto con gli editori è complesso. Avendo a che fare con un sistema economico e industriale non sempre le finalità coincidono perfettamente. Solo poche volte ho collaborato con grafici professionisti, non perché non lo volessi fare ma perché non è possibile far gestire un progetto su cui hai lavorato per anni a qualcuno che senza saperne nulla lo impagina in una settimana, casomai seguendo le mode del momento. Assistiamo a una sovrapproduzione di libri 
d'arte e fotografici in cui a volte il grafico è più interessante dell'autore. Ai giovani consiglio di essere parchi con gli effetti grafici, il rischio è che ci si ricordi solo di quelli e non del contenuto del libro. So di non venire ascoltato ma hanno comunque tutta la mia stima. Ho invidiato moltissimo Aby Warburg apprendendo che quando si dissociò economicamente dalla famiglia di banchieri lasciò tutte le banche al fratello, chiedendogli però le risorse per poter comprare tutti i libri che desiderava.

V. T.: Cosa è importante quando lei compone un libro con le sue immagini?

O. B.: La grafica non deve incombere, deve essere invisibile o al servizio delle immagini. Ci sono comunque eccezioni, la grafica può essere invasiva, se è integrata all'oggetto. Ho fatto libri con una spirale che spezza il centro dell'immagine, altri dove l'immagine è divisa in due con una striscia bianca al centro, per eliminare la piega del libro. Presentando un nuovo progetto alla mia galleria di New York avevo preparato un leporello che avrei voluto mettere in una bacheca, era l'unico modo per rendere visibile la sequenza, lo sviluppo e la totalità delle opere (non tutte potevano essere esposte per le grandi dimensioni) in un unico colpo d'occhio. La gallerista non decise di fare la bacheca, ma di farne un libro. A volte i libri nascono per caso. La sequenza deve essere possibilmente di acciaio.

M. M.: Cosa intende per sequenza «d'acciaio»? Che legami devono sussistere tra un'immagine e l'altra? Di tipo narrativo, o si tratta di un montaggio, che è flusso, ma che è anche il bianco (o il nero) tra un'immagine e l'altra?

O. B.: Prediligo libri semplici, le immagini sono di difficile lettura e hanno bisogno di chiarezza. Anche le immagini apparentemente semplici e belle hanno bisogno di tempo e rigore di presentazione. Il fatto che siano semplici e belle dovrebbe essere motivo di sospetto, non vuol dire infatti che siano vuote, vanno scrutate con molta attenzione. Non sappiamo perché siamo attratti dalle immagini. Per sequenza «d'acciaio» intendo il pensiero che sorregge e rende leggibile il progetto. Naturalmente ogni libro fa storia a parte, non c'è una regola aurea per la sequenza «d'acciaio». La sequenza «d'acciaio» è la regola aurea. Non ho mai usato, per mia scelta pagine nere o colorate. Non metto mai volentieri più di un'immagine per pagina. Anche quando si affiancano due immagini, una per pagina, deve esserci un motivo per farlo.

V. T.: Sempre rispetto alla sequenzialità delle immagini e aprendo il discorso alla dimensione cinematografica, il fotografo Jeff Wall ha affermato di seguire Roland Barthes nel vedere il cinema come una sequenza di immagini fisse. Come vive la visione di un film?

O. B.: Nei film non seguo sempre e in modo lineare lo svolgimento della storia perché mi perdo nelle immagini. Le storie spesso sono più prevedibili delle immagini. Mi accade lo stesso con le canzoni, il significato delle parole 
non lo seguo molto. In aereo guardo i film senza audio, gli auricolari e le cuffie dopo un po' mi infastidiscono. Non sono mai riuscito a vedere tutto intero seguendone con cura la trama, quello che a suo tempo era un film di culto come Il cielo sopra Berlino di Wim Wenders. Non credo che il cinema e la fotografia siano linguaggi assimilabili, uno si muove per poter essere, l'altro è immobile per poter essere.

M. M.: Cosa pensa di Wenders fotografo?

O. B.: Di Wim Wenders mi piacciono particolarmente le fotografie che fece ad Akira Kurosawa.

M. M.: Ci sono diverse sue affermazioni sulla letterarizzazione o fantasmatizzazione della realtà, secondo le quali non avremmo a che fare con una realtà vergine o nuda, ma sempre popolata di immagini e di parole. Fino a che punto si spinge questa irrealtà? Viviamo in una realtà di segni che anticipano qualsiasi esperienza, o è possibile secondo lei un'esperienza effettivamente nuova? La fotografia può contribuire a fondarla?

O. B.: Nel 1982, quando vidi Blade Runner, ne fui molto colpito. Anni dopo a Hong Kong prima, poi a Shanghai a Pechino o a Dubai mi resi conto che via Philip Dick, Ridley Scott aveva previsto l'iconografia della città del futuro.

Pochi giorni fa nel 202I nel Capitol Building a Washington è "accaduta" la continuazione del finale di Joker (2019) di Todd Phillips. I tempi si accorciano.

Se non ci fosse stato un libro come Robinson Crusoe, tantissimi viaggiatori temporaneamente sperduti non avrebbero potuto attingere a un codice di comportamento a loro famigliare.

Anche l'inferno, il paradiso e il purgatorio, se non ci fosse Dante, come ce li immagineremmo?

Credo che esista solo la letteratura. L'unico modo per dare vita alla realtà è sintetizzarla in una forma artistica che può essere la letteratura, oppure l'immagine. Quello che resta è un'immagine o un racconto. Il passato non c'è mai stato.

Un'esperienza nuova potrà essere l'ormai imminente stanziamento umano su altri pianeti. Quanto del tanto fantasticato dalla fantascienza contribuirà alla progettazione di lontanissimi nuclei abitativi futuri? Quanto esiste già di quel mondo nuovo? Come si apprende da Ludovico Ariosto i nostri neuroni viaggiano nello spazio da secoli.

La fotografia non so se esisterà ancora nel lasso di tempo che la separa dalla fondazione di un'esperienza inedita del mondo. Fra non molto sicuramente basterà pensarla, un'immagine, per vederla e condividerla. 


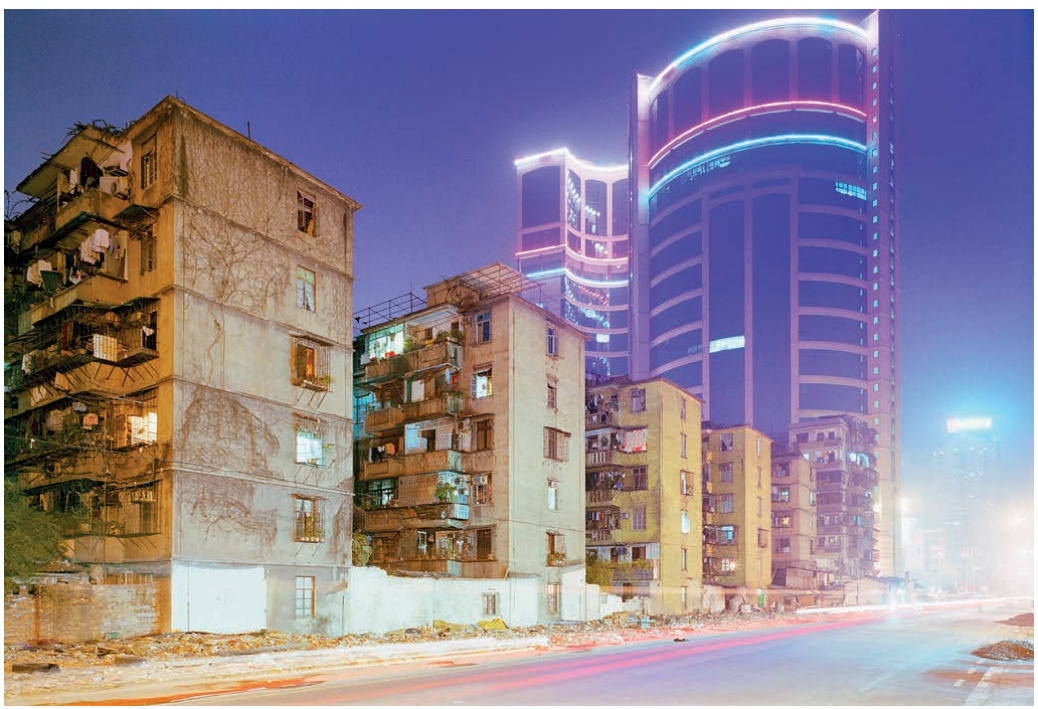

Fig. 3: Olivo Barbieri, Canton, Cina, I998.

V. T.: La sua insistenza sull'importanza di parlare di immagini e non di fotografie si lega alla presenza, nelle sue opere, di un'interrogazione sui confini tra realtà e rappresentazione, verità e finzione?

O. B.: Il problema della fotografia è che, a differenza della letteratura, non ha avuto dipartimenti universitari che l'abbiano studiata, sviscerata, classificata, definita. Non c'è ancora un teorico autorevole che abbia detto delle cose fondamentali sulla fotografia.

La quantità di letteratura di qualità che c'è sugli scrittori, i musicisti e gli artisti è rilevante, sui fotografi ce n'è poca e in buona parte solo agiografica.

Le storie della fotografia sono state scritte sovente da fotoamatori, che amavano moltissimo la fotografia, molti erano o avrebbero voluto essere fotografi. Hanno però semplicemente elencato cronologicamente le gesta dei fotografi. Parallelamente ci sono state le varie storie sociali della fotografia...

Poi il digitale. Quando la prima immagine è stata digitalizzata, e comunicata attraverso una mail, lì si è rotto l'incantesimo e la fotografia è evaporata.

Le fotografie, ormai, come tutte le immagini, sono immateriali. Il teletrasporto, rendendole universalmente fruibili e di conseguenza trasformabili, le ha mutate di segno.

Alla luce di questa evaporazione ho deciso che mi interessavano le immagini, perché più comprensive delle possibilità della rappresentazione.

M. M.: A proposito di immagini, un recente sviluppo della sua produzione sono le sculture ispirate ai codici cifrati di nomadi e vagabondi, segni 
sospesi tra la realtà e la leggenda urbana che lei trasforma in monumenti. $\grave{E}$ in cerca di altre lingue oltre quella della scrittura alfabetica?

O. B.: Nel 2018 al MoMA di New York c'era la mostra Constantin Brâncuşi Sculpture. Nel sito del museo si poteva vedere un film in bianco e nero (ho sempre ammirato Man Ray e Constantin Brâncuşi perché capendo che i fotografi non erano in grado di fotografare le loro opere decisero di farlo da soli) girato da Brâncuşi tenendo una cinepresa a mano libera. Questo filmato mi colpì molto, per la libertà con cui era girato e per il soggetto che riprendeva. Mostrava una torre infinita appena terminata (I938), alta quasi trenta metri, al centro di un parco a Târgu Jiu in Romania. Non sapevo esistesse una torre infinita così grande, conoscevo solo quelle nell'atelier dello scultore custodito al Centre Pompidou a Parigi e poche altre. Târgu Jiu è vicinissimo a Hobita, paese natale di Brâncuşi. Tutta la regione è costellata di false torri infinite. Durante la primavera-estate del 2018 sono andato in Romania tre volte per fare fotografie. Avevo appena terminato un paio di maquette per il libro che intendevo pubblicare. Documentandomi su quei luoghi ho ripensato alla simbologia attribuita ai rom e agli hobos americani. A quell'alfabeto di segni che vengono trovati sulle case, che danno indicazioni sulla possibilità di avere o prendere qualcosa. Una città invisibile nella città. Non si sa se l'uso di questi segni sia reale o se sia una leggenda metropolitana, ma l'alfabeto esiste. Partendo da questi simboli, ricopiandoli e riportandone con una scritta il significato («There are only women», «Alcohol in this town», «Man with a gun lives here», «Religious talk will get you a meal here» ecc.) ho realizzato dei disegni. Poi dei disegni tridimensionali e in seguito una dozzina di prototipi in legno e ferro di circa trenta centimetri con la scritta incisa alla base.

In quei giorni stavo preparando una mostra su un apparente paradosso. Molti dei luoghi che consideriamo naturali come le Alpi, le Dolomiti, le montagne sacre cinesi, l'isola e il mare di Capri, la costiera adriatica e le grandi cascate sopravvivono grazie al turismo, che in un certo qual modo produce l'economia per conservarle. In definitiva molti luoghi che consideriamo naturali funzionano come parchi tematici.

Le immagini di quattro discariche abitate da migliaia di persone e animali nel sud est asiatico, enormi parchi a tema del riciclo e dell'intossicazione (specularmente, ogni negativo contiene le stesse informazioni di un positivo), chiudevano il cerchio.

Quando il curatore della mostra vide i prototipi delle sculture fu incuriosito e mi incoraggiò a produrle in grandi dimensioni. Era il parco tematico esoterico che mancava, un prodromo di simboli astratti che funzionano come gli algoritmi invisibili e comprensibili per pochi, che ci dividono a nostra insaputa in gruppi (parchi tematici dell'esistenza) per età, sesso, razza, status sociale ecc. 


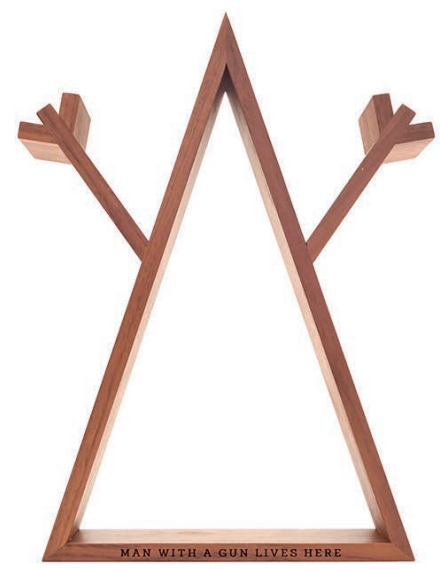

Fig. 4: Olivo Barbieri, Man With A Gun Lives Here, 2018.

V. T.: Ne L’impero dei segni Barthes percorre lo spazio giapponese come se fosse un grande testo; costruendo un'ambivalenza tra i segni europei e i segni orientali, anche rispetto al modo di leggere e orientarsi nello spazio. Il suo scatto del I980 Casa del caso attesta il riconoscimento nella realtà di forme di testualità; un aspetto che fa parte anche della storia della fotografia, pensando a Walker Evans o Ralph Steiner. C'è per lei una testualità della realtà verso cui il fotografo deve "solo" essere ricettivo, piuttosto che costruirla?

O. B.: Per Ralph Steiner e Walker Evans la fascinazione dipendeva forse quasi esclusivamente dal fatto che fossero i primi testimoni di questo fenomeno (le grandi, e piccole insegne) della modernità. Un nuovo modo di promuovere merci e servizi.

Parole nel paesaggio e nelle città, non solo dipinte ma anche tridimensionali e al neon, leggibili di notte. Una delle fotografie più misteriose di Walker Evans è Workers Loading Neon "Damaged" Sign into Truck, West Eleventh Street, New York City 1928-30.

Ho ritrovato lo stesso stupore in Cina, dove c'era ancora un uso estremamente disinvolto e massiccio di insegne e cartellonistica. Le cose però stanno cambiando, a Pechino sembra che le più invasive le toglieranno e addirittura gestiranno tutta l'illuminazione pubblica secondo un piano di riordino coordinato. Ero molto interessato alla testualità degli ideogrammi. Parole nel paesaggio, disegni. In Giappone c'è una teoria controversa, ma suggestiva, secondo la quale la scrittura nasce dalla pittura, dalle immagini.

Seguo da anni l'opera di Edward Ruscha, che da più di mezzo secolo partendo dalla cartellonistica stradale americana produce opere di quasi 
solo parole, spesso nel paesaggio. Ha addirittura inventato un font e rifiutando l'uso dell'informatica fa ancora tutto manualmente.

Ricordo che per un'ora cercai invano di spiegare, a un critico d'arte tedesco, il gioco di parole sulla facciata di una casa di campagna «la casa del caso».

Werner Herzog sosteneva che le storie nascono dai paesaggi.

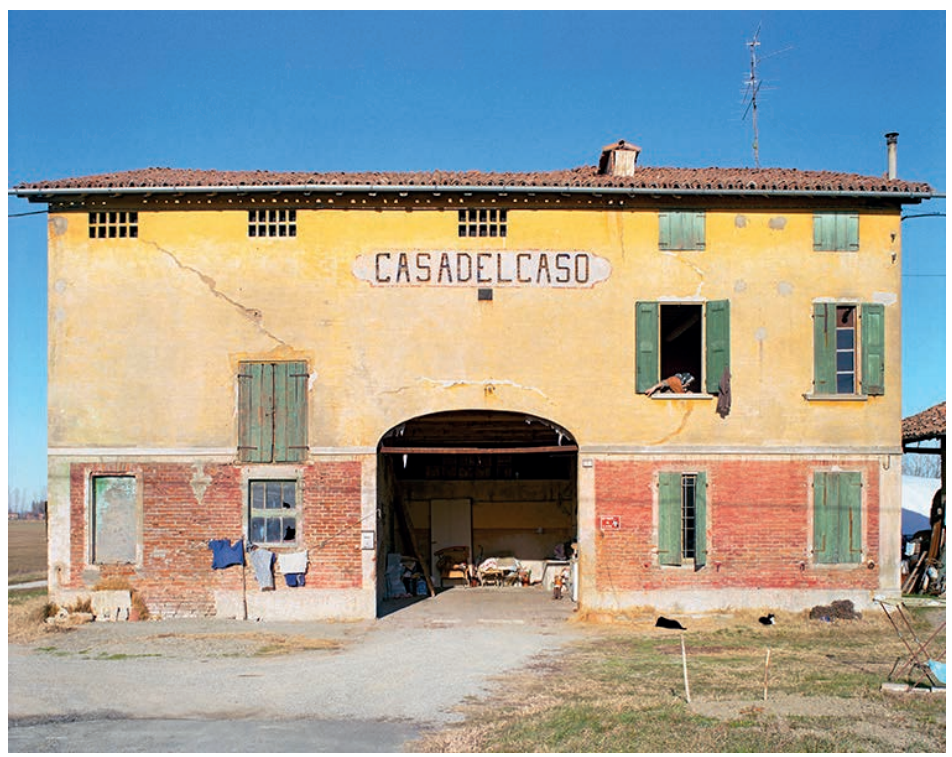

Fig. 5: Olivo Barbieri, Emilia Romagna, 1980.

M. M.: In alcune sue immagini lei include il titolo all'interno della fotografia, come in Hanging Ideograms, Pudong Shanghai, China 20I4, in cui il titolo è integrato come una didascalia su fondo nero nella fotografia stessa. Che cosa ha voluto dire?

O. B.: Quello che ho rappresentato è un ideogramma sospeso enorme, sul tetto di un palazzo di novantacinque piani a Shanghai.

A fine anni ottanta ero stato a Datong (Shanxi) in treno, impiegandoci da Pechino una notte. A Datong vidi un tempio sospeso. Appeso alla roccia della montagna all'altezza di settantacinque metri sul fiume per evitare che i testi sacri venissero danneggiati dalle frequenti invasioni e alluvioni. Vi convissero dal sesto secolo dopo Cristo pacificamente le tre religioni tradizionali cinesi: buddismo, taoismo e confucianesimo.

Nel 20I4 ritornai a Datong, impiegandoci in treno poco più di 2 ore e lo fotografai. Mi interessava mettere in relazione questa necessità di sospendere grandi oggetti, non necessariamente indispensabili. 
Per stabilire una relazione tra i due soggetti inserii le rispettive didascalie in calce alle immagini: Hanging Ideograms, Shanghai, China 2014 e Hengshang Hanging Temple, Datong, China 2014.

Le didascalie sono importanti, sono l'opera. Devono essere semplici. Luogo e data sono indispensabili. Guardando una fotografia penso sempre a come si sentiva, chi l'ha scattata. Penso a chi era che stava fisicamente davanti all'immagine. Quando si guardano le opere di Diane Arbus, non si riesce a non vederla mentre fotografa. Una fotografia è qualcuno che capisce qualcosa nel frammento temporale in cui capisce.

Ecco perché ho un po' di difficoltà con i droni. Fotografare con un elicottero è essere lì e vedere da quel punto di vista. Vedere il mondo da un punto di vista dove non sei fisicamente in quel momento è problematico ma è anche molto interessante. I punti di vista prevedibili non sono più imprescindibili.

Si ha più a che spartire con gli interrogativi sollevati dalla teoria dei quanti che con le vecchie regole della prospettiva. Dobbiamo entrare in un altro mondo, siamo ormai in un mondo nuovo. Un punto di vista solo non esiste più. Qualcuno o qualcosa che riprende un'immagine da un punto di vista senza esserci, e la costruisce, la definisce da un altro punto di vista, è una possibilità molto interessante di cui bisogna fare tesoro. Nella nostra quotidianità siamo immersi nelle immagini e da quanto reso possibile dal calcolo quantistico. Non sappiamo nulla delle immagini e nulla dei quanti. Li vediamo soltanto funzionare.

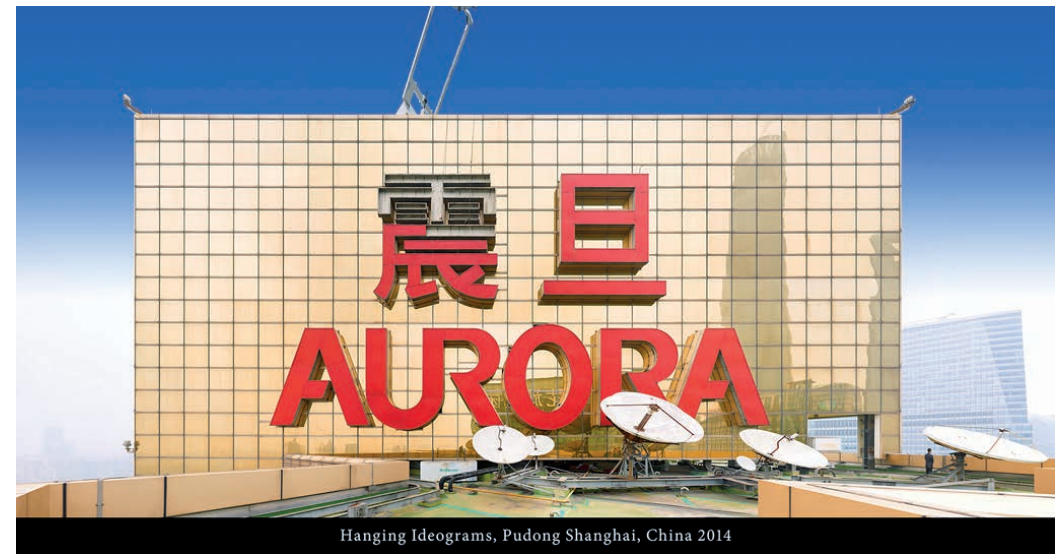

Fig. 6: Olivo Barbieri, Shanghai, Cina, 2014.

M. M.: Mi ricollego a questo e al problema della distanza, che lei ha già evocato a proposito della possibilità di vedere gli Stati Uniti soltanto spostandosi in Cina o con l'uso dell'elicottero e adesso l'uso del drone, o con la montagna come punto di vantaggio per l'osservazione dall'alto. È un aspet- 
to che può essere messo in rapporto anche con il fatto che la distanza non ci fa sentire le voci dei luoghi. Un tema che era stato evocato da Vaccari ai suoi esordi e che poi anche lei ha ripreso. Questo distanzia la fotografia dalla parola, almeno in quanto suono, in quanto espressione vocale.

O. B.: site specific_ è un progetto globale che ridefinisce la forma della città contemporanea. Le immagini del progetto site specific_, iniziato nel 2003 , sono tutte realizzate dall'elicottero. Ho sorvolato più di cinquanta città, metropoli e megalopoli. Adottare una postazione flottante ha limitato il rischio di visioni obbligate e prevedibili. Dopo l'undici di settembre e la distruzione delle torri gemelle mi sono chiesto che valore reale hanno oggetti architettonici identitari come il Colosseo, la torre di Pisa, il Tower Bridge, Time Square ecc. Se sparissero, come muterebbe la condivisa percezione psicogeografica del mondo? Ho tentato di rileggere la città contemporanea come fosse un plastico in scala, una installazione temporanea, e da un punto di vista scollegato dalle parole, dai suoni, dagli odori. Immagini e parole possono essere separate, a volte dovrebbero esserlo. Le fotografie sono una messa in codice dell'immagine del reale, le parole sono una messa in codice dello scheletro dei pensieri.

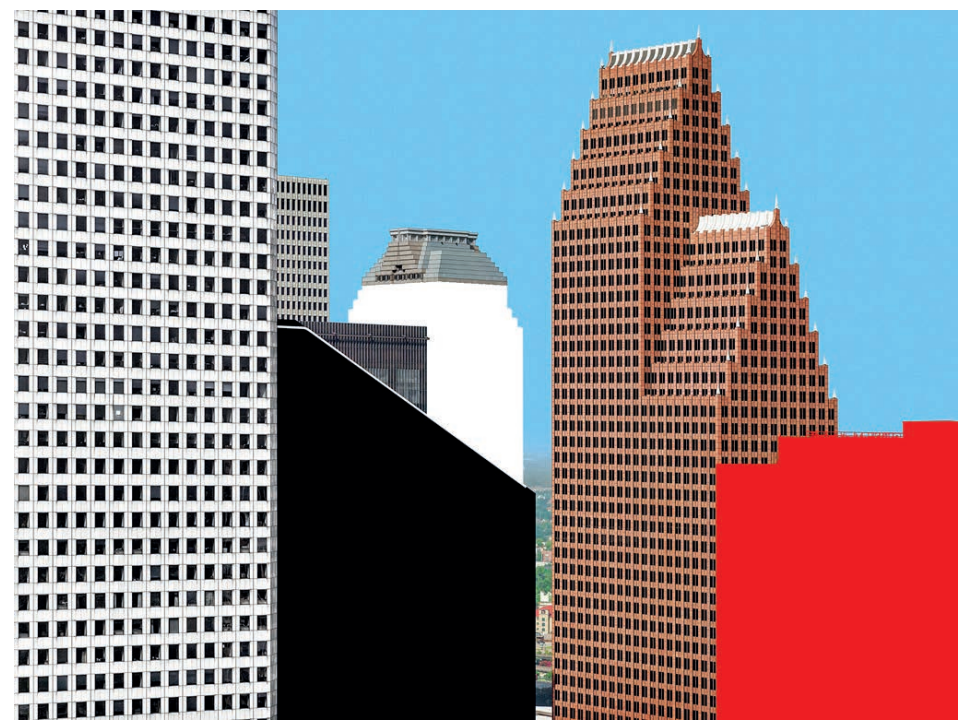

Fig. 7: Olivo Barbieri, site specific_HOUSTON I2, 2012.

V. T.: In merito alla presenza-assenza della parola, si cita spesso la dimensione narrativa dell'immagine, la possibilità che l'immagine costruisca delle storie, anche in virtù di quello che non è contenuto nella cornice della 
fotografia. Per lei conta la dimensione narrativa di un'immagine e delle sue immagini in particolare?

O. B.: John Szarkowski, per quasi trent'anni Director of photography del Museum of Modern Art di New York, anni in cui ha ridefinito la storia della fotografia americana, consigliava di fare attenzione ai bordi della fotografia e di tener conto che sovente quanto sta fuori dall'immagine è più importante di quanto rappresentato nel rettangolo della fotografia.

Sarebbe a dire che ciò che non vediamo, non sappiamo, è più importante di ciò che crediamo di vedere sapere.

Gordianamente credo che le parole facciano le parole e le immagini facciano le immagini.

La soluzione sta altrove. Eminenti dantisti sostengono che non si sa che cosa sia la poesia e che Dante rimane un mistero; lo stesso per le immagini, non sappiamo perché, ma non possiamo farne a meno!

A fine anni settanta c'era un dibattito forsennato: ma questa fotografia è narrativa o non è narrativa? Non ho mai capito se essere narrativa fosse un valore aggiunto o un difetto.

V. T.: Thomas Ruff ha affermato che la fotografia può riprodurre solo la superficie delle cose, mentre altri credono che la fotografia possa cogliere anche un oltre della superficie.

O. B.: Credo che Thomas Ruff abbia ragione, la fotografia galleggia sull'immagine del reale. Ciò non toglie che non si possano produrre immagini che vadano in profondità. Purtroppo il dibattito sulla fotografia è avanzato considerandone quasi solo le implicazioni sociali e politiche. Anche la storia della fotografia così come è scritta è diventata ingombrante, tanti autori riprendendo vecchie tecniche o stilemi, la girano e rigirano, come una volta si rigirava la stoffa dei vecchi cappotti. Lo stupore della prima immagine apparsa in camera oscura, per decenni e decenni, ha sostituito lo stupore del mondo.

V. T.: Come si pone rispetto alla diffusione delle immagini sui social, come ad esempio Instagram, dove lei stesso ha un profilo? Qual è per lei la funzione di queste piattaforme?

O. B.: Volevo tenermi fuori dai social, comportano una perdita di tempo colossale. Richiedono una frequentazione ininterrotta. I social sono però uno strumento formidabile per misurare la temperatura dello spirito del tempo.

Conosco poco Instagram, in Facebook non sono mai entrato. Me ne servo esclusivamente per comunicare l'uscita di un libro, l'apertura di una mostra e poco altro. Comunico credo con un ristretto gruppo di persone che hanno interessi simili ai miei. Ciò che dopo un po' stanca è il rigido confine del parco a tema telematico, contro il quale come in un videogioco si rimbalza tra algoritmi neanche tanto invisibili. All'inizio ti sembra che si sia 
allargato il cortile, dopo un po' capisci che il cortile è stato sostituito, e che forse se lo meritava.

V. T.: Pensa che sul web le immagini in qualche modo si snaturino e fa parte del gioco, oppure secondo lei permane un'essenza dell'immagine? Nel momento in cui posta un'immagine che percezione ha del porto in cui approda?

O. B.: Stiamo vivendo in un'epoca in cui facciamo molta ginnastica per praticare uno sport sconosciuto, ci stiamo allenando per praticare uno sport di cui non sappiamo quali regole avrà. Tutto questo farneticare istantaneo porterà alla ridefinizione di quelli che una volta erano gli strumenti del comunicare, che ora sembrano più una gelatina ectoplasmica falso-panottica. Alcuni sostengono che grazie ai nuovi media e a nuove app esoteriche, i giovani stiano progettando inediti modelli di democrazie in cui finalmente potranno vivere. Un mondo parallelo ma reale, funzionante tramite la blockchain technology. Anche le immagini muteranno, indicizzate dalla blockchain technology. Ora quando posto un'immagine non credo accada nulla di rilevante, forse perde un po' d'aura in senso antico. Come muterà la nostra percezione attraversata dalle criptoimmagini?

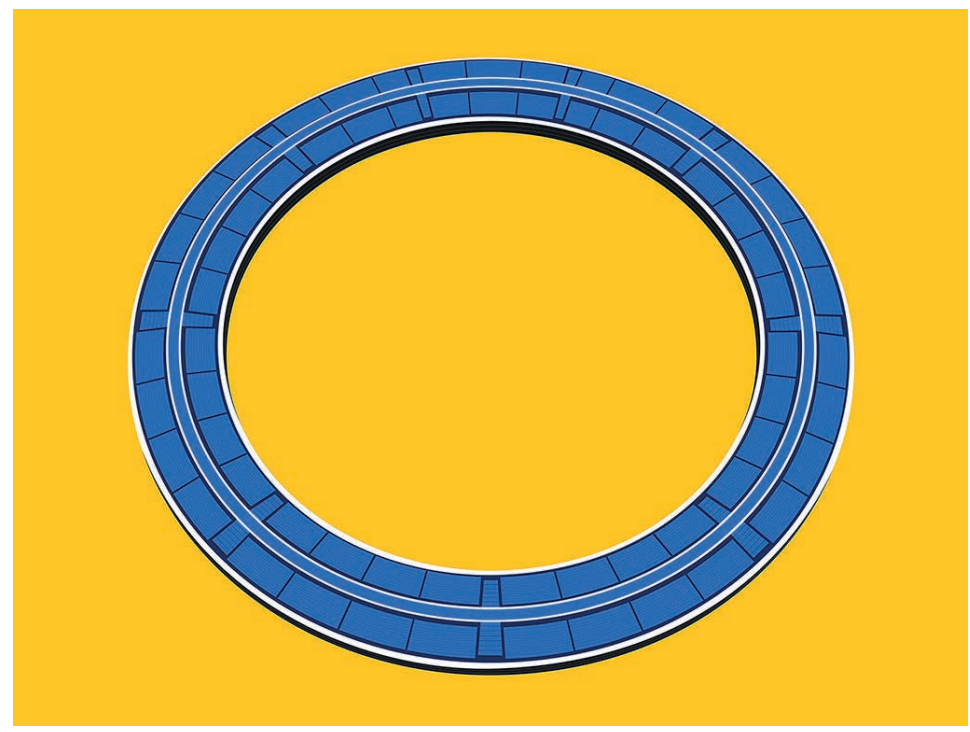

Fig. 8: Olivo Barbieri, Apple Park Cupertino CA, 2017.

V. T.: Lei è anche autore di video. Che rapporto c'è per lei tra il binario dell'immagine fissa e dell'immagine in movimento? 
O. B.: Sono binari paralleli. Sono linguaggi diversi. Ciò non toglie che con linguaggi e codici diversi si possano trarre le stesse conclusioni e analizzare gli stessi problemi e sviscerare le stesse suggestioni. Sono tecniche o tecnologie che non sono assimilabili, sono sistemi di pensiero completamente autonomi. Uno è legato alla contemplazione, l'altro al movimento.

M. M.: Riprendendo il riferimento iniziale relativo al rapporto con la letteratura, quali sono le sue letture attuali?

O. B.: Leggo sempre meno narrativa. Recentemente ho letto alcuni saggi di scienze cognitive e nuovi studi su LSD e percezione. Come tutti sto cercando di capire la meccanica quantistica che è alla base del funzionamento della blockchain technology. Per i tempi del lockdown, ma deve essere lockdown totale, può essere utile la lettura di Casa di foglie di Mark Z. Danielewski. 
